\title{
Application of fiber optic FBG techniques in analysis of strain in engineering machines
}

\author{
Janusz Juraszek \\ University of Bielsko-Biała, Poland
}

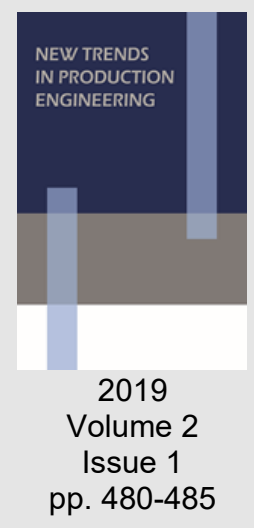

Date of submission to the Editor: 05/2019

Date of acceptance by the Editor: 08/2019

\section{INTRODUCTION}

Optical sensors with fiber Bragg grating (FBG) enable mainly very precise strain measurement. What is important, they do not require calibration because they come factory-calibrated. The development of fiber optic techniques made it possible to monitor such parameters as: temperature, displacement, pressure, acceleration and even humidity. The author has been conducting strain research using fiber Bragg gratings since 2012. For the first three years (2012-2014) they were carried out in the framework of the European project. Further research concerned the analysis of strain of overhead cranes, mining ropes, geogrids used to reinforce the subsoil for roads, strain of power lines, bridges, footbridges. concrete surfaces or even monitoring the strain of a residential building (Juraszek, 2018a, 2018b). The results of the research were documented in papers. Studies of high-load bodies of engineering machines have not been published so far. An interesting method of soil strain analysis was presented in paper (Runzhou et al., 2019), where a method of soil strain measurement was based on fiber Bragg grating (FBG) sensing technology. Other interesting application (Boateng et al., 2019) are different capillary tubes assessed as isolation materials for FBG sensors for composites cure monitoring.

\section{FBG STRAIN SENSOR}

The FBG sensor is produced by the formation of a single-mode optical fiber. It is obtained by applying a periodic pattern of intense ultraviolet light on the core of single-mode optical fiber. The wavelength is approx. $300 \mathrm{~nm}$. Specially selected radiation force with such a wavelength causes a slight "destruction" of the optical fiber - breaking of some of the silicon-oxygen bonds in the polymer chain. The result of this is a slight increase in the refractive index in the area where UV light was applied. The periodic structure is created by exposing the fiber core to two beams of light, placed at right angles to each other. This results in their overlapping, and thus a change in the intensity of light in the area that is exposed to these very beams. Once the cuts are applied to the fiber, it is coated 
and the coating curing stage follows. The length of the Bragg sensor used for geosynthetic materials testing was approx. $8 \mathrm{~mm}$.

At each periodic change in the direction of the wave propagation, a small amount of light is reflected. Reflected from the incisions, light rays overlap when the difference in reflected rays, from two parallel planes, is equal to a multiple of the length of the incident wave. This is determined by the Bragg condition, and the wavelength at which such reflection takes place is called the Bragg wave. Light signals at wavelengths other than Bragg wavelength are transparent.

The bandwidth of the light beam reflected by the grating is $0.2 \mathrm{~nm}$, and the Bragg wavelength depends on the grating period produced in the optical fiber. The optical fiber distortions, for a given temperature, affect directly the grating period and consequently the length of the reflected light wave.

Optical fiber distortion can also occur as a result of changes caused by the thermal expansion of the optical fiber material. The change in wavelength caused by this factor is expressed by dependence:

$$
\begin{aligned}
& \Delta \lambda_{B}=s_{\varepsilon} \cdot \Delta \varepsilon+s_{T} \cdot \Delta T \\
& s_{\varepsilon}=\lambda_{B}\left(1-r_{a}\right) \\
& s_{T}=\lambda_{B}(a+\xi)
\end{aligned}
$$

where:

$\Delta \varepsilon-$ strain change,

$\Delta T$ - temperature change,

$s_{\varepsilon}$ - strain value, for a wavelength range of $1520-1560 \mathrm{~nm}$ equal to $1.2 \mathrm{pm} / \mu \varepsilon$,

$r_{a}$ - elongation constant,

$s_{t}$ - temperature value, for a wavelength range of $1520-1560 \mathrm{~nm}$ equal to 1.2 $\mathrm{pm} / \mu \varepsilon$,

$a-$ linear temperature coefficient,

$\xi$ - temperature constant.

Principle of operation of the FBG sensor is shown in Figure 1.
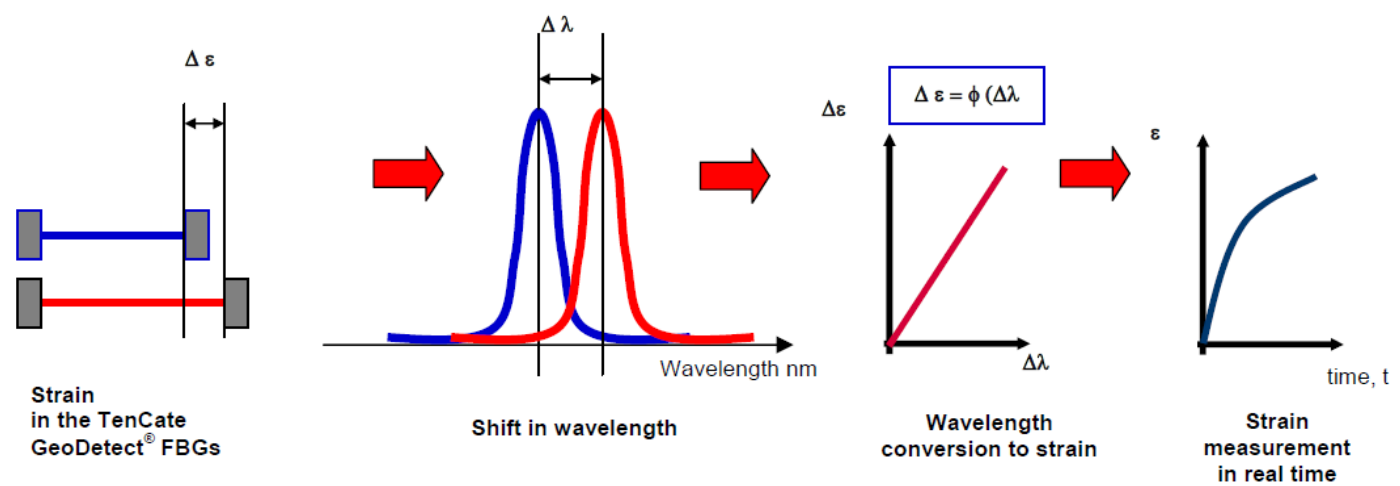

Source: (www.geosynthetica.com)

Fig. 1 Principle of operation of the FBG sensor

The FBG devices used to for diagnostics include:

o Optical interrogator - optoelectric device for supplying light to the fiber optic system, reading the wavelength shift and summing the signals and sending them to the recorder, 
o Optical fiber Bragg grating - optical fiber section, inside which Bragg grating has been applied, causing the change in the refractive index,

o Temperature compensator - compensates for the change in Bragg wavelength when measured at temperatures other than $20^{\circ} \mathrm{C}$,

o Processing and analysing unit - a recorder connected to software that allows to observe the changes in the analysed values in real time.

Two types of FB sensors were used in conducted experiments on geosynthetics. The first of these is shown in Figure 2. The FBG sensors are located in the central part of the sensor. Each of them, including the place for gluing, occupies $25 \mathrm{~mm}$. The sensor is terminated with two FC/APC connectors allowing connection of the telecommunications fiber optic with the optical interrogator.

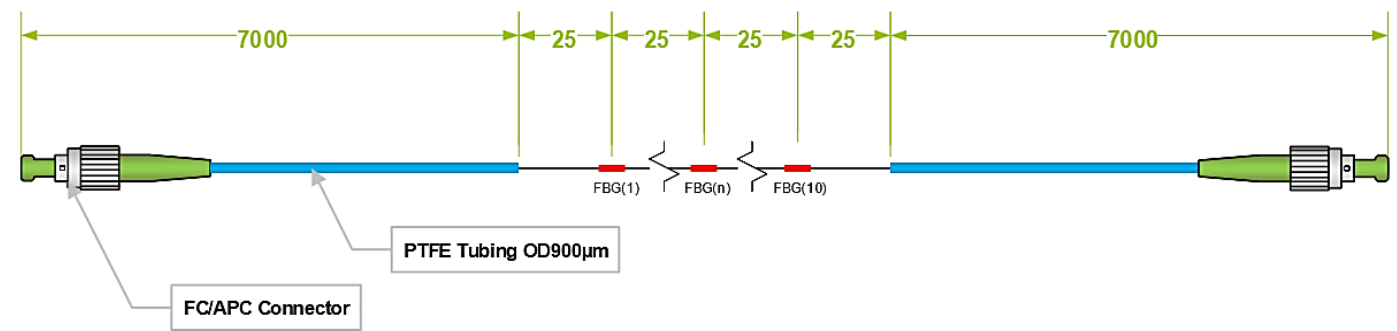

Source: (sylex.sk)

Fig. 2 Diagram of the F-01 sensor

The sensor technical parameters are listed in Table. 1.

Table 1 Parameters of F-01 strain sensor

\begin{tabular}{|c|c|c|}
\hline Performance properties & & \\
\hline Strain range & \multicolumn{2}{|c|}{ $\pm 3000 \mu \varepsilon$} \\
\hline Accuracy (guaranteed) & \multicolumn{2}{|c|}{$<0.3 \% \mathrm{FS}$} \\
\hline Accuracy (typical) & \multicolumn{2}{|c|}{$<0.25 \% \mathrm{FS}$} \\
\hline Precision (guaranteed) & \multicolumn{2}{|c|}{$<0.15 \% \mathrm{FS}$} \\
\hline Precision (typical) & \multicolumn{2}{|c|}{$<0.10 \% \mathrm{FS}$} \\
\hline Temperature compensation & \multicolumn{2}{|c|}{ External } \\
\hline Operating Temperature range & \multicolumn{2}{|c|}{$-20^{\circ} \mathrm{C}$ to $+80^{\circ} \mathrm{C}$} \\
\hline Ingress Protection rating & \multicolumn{2}{|c|}{ N/A } \\
\hline Grating properties & Typical (standard) & Customizable \\
\hline FBG wavelength range & $1510-1590 \mathrm{~nm}$ & $1460-1640 \mathrm{~nm}$ \\
\hline Nominal (central) wavelength tolerance & $\pm 200 \mathrm{pm}$ & -- \\
\hline Reflectivity & around $40 \%$ & $1-99 \%$ \\
\hline FWHM between (3dB bandwidth) & $450-550 \mathrm{pm}$ & $0,1-$ few $\mathrm{nm}$ \\
\hline SLSR (side lobe suppression) & $>15 \mathrm{~dB}$ & up to $20 \mathrm{~dB}$ \\
\hline PDFS (polarization depended WL shift) & $<5 \mathrm{pm}$ & -- \\
\hline FBG length & $<5 \mathrm{~mm}$ & $5-12 \mathrm{~mm}$ \\
\hline Fiber coating & Polyimide & Ormocer, others \\
\hline Fiber type & Bend insensitive & Pure silica \\
\hline
\end{tabular}

\section{Physical properties}

Active FBG element length ${ }^{1)}$

Active gluing length

Fiber Bend Radius

Connector type ${ }^{2)}$

Mounting method

$5-12 \mathrm{~mm}$

Around $30 \mathrm{~mm}$

$10 \mathrm{~mm}$

FC/APC

Direct surface gluing

Source: (sylex.sk) 
This system has many advantages that distinguish it from classic strain gauges. Using the appropriate configuration of the test equipment, it is possible to analyse strains in as many as 1200 measuring points, using one optical fiber and a measuring set. Fiber optic sensor instrumentation consists of an optoelectronic interrogator and software, thanks to which the measuring system is adapted to the needs of a given application, achieving measurement accuracy at a level of $\Delta \varepsilon=10^{-6}$ (microstrain).

\section{RESULTS OF STRAIN TESTS OF RNGINEERING MACHINE BODY}

The body of the engineering machine consists of two highly loaded columns. Each of them transfers a load of about 400T. It has been proposed to monitor the strain of each column, both its internal and external surface. The fiber optic sensors have been mechanically mounted to the columns using a mechanical system that has been registered as a patent claim. An important issue is pretensioning of a fiber optic sensor. The level of 1000 strain was adopted. This allows the analysis of both stretching and compression of the columns. The measuring system consisted of a two-channel optical interrogator and a computer recorder with appropriate software. The fixing points for strain sensors are shown in Figure 3.

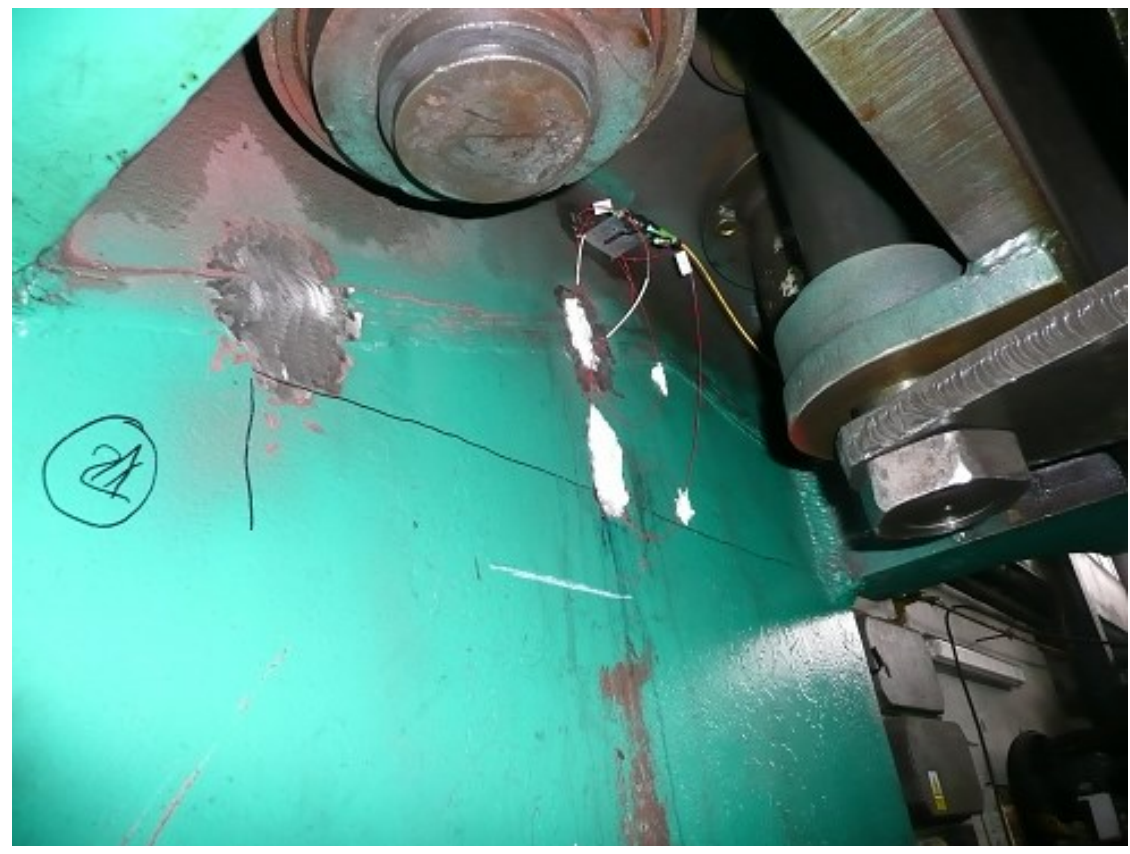

Fig. 3. Location for the strain sensors

The results of the strain tests of the engineering machine columns are shown in Figures 4 and 5. They depict the following strains:

The right column on the outside $\mathrm{S1}$

The right on the oinside S2

The left column on the inside S3

The left columns on the inner side on the weld S4

The left column on the outside S5. 
The highest strains were recorded for the right column on the outer side and amounted to 270 strain. Other sensors recorded strains at a much lower level of 135 to 150 strain. A thorough analysis of the outer surface of the right column discovered the initial phase of cracking. The research concerned the soil reinforced with a geogrid - Figure 4 .

The research has enabled the detection of serious damage that could have caused a major accident or even a catastrophe.

Strain studies were also carried out for the classic subsoil. The results of the tests are presented in Figure 5 . This caused a reduction in strain values by about 50 microstrain

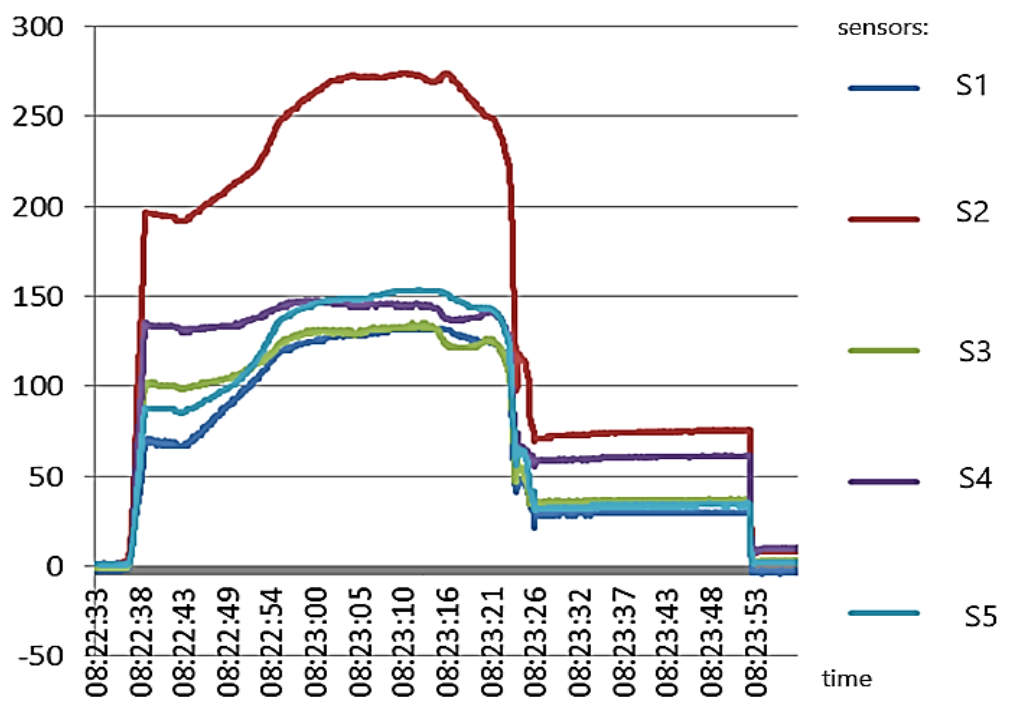

Fig. 4 Strain of the engineering machine collar - the subsoil reinforced with a geogrid

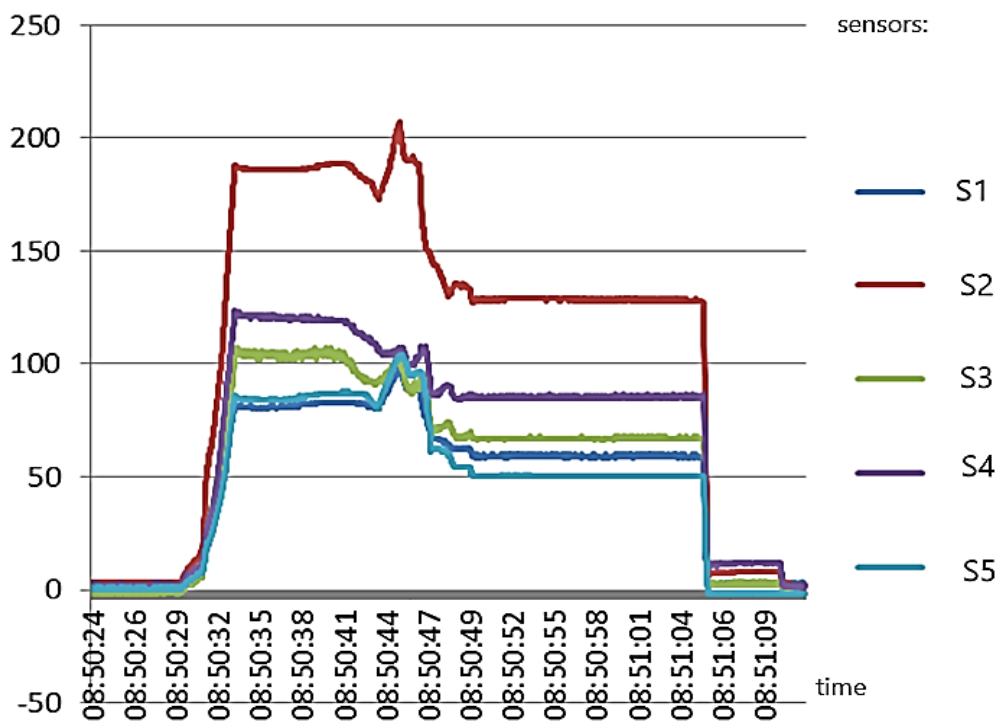

Fig. 5 Strain of the columns of the engineering machine, classic subsoil

\section{CONCLUSION}

1. The use of strain sensors enables precise measurement of deformations in difficult and dangerous mining conditions.

2. The strain monitoring of engineering machines enables early detection of cracks and other damage. 
3. The FBG method is also distinguished by significant operational durability (no time and temperature drift occurs).

\title{
REFERENCES
}

Juraszek J., (2018a): Hoisting machine brake linkage strain analysis. Archives of Mining Sciences vol. 63, no. 3, pp. 583-597

Juraszek J., (2018b): Strain and force measurement in wire guide. Archives of Mining Sciences vol. 63, no. 2, pp. 321-334

Runzhou Y. el al., (2019): A novel fiber Bragg grating (FBG) soil strain sensor. Measurement. Volume 139, Pages 85-91

Boateng E.K.G., Schubelb P., Umer R, (2019):Thermal isolation of FBG optical fibre sensors for composite cure monitoring Sensors and Actuators A: Physical Volume No 287, Pages 158-167.

\begin{abstract}
.
The paper presents the use of fiber optic FBG strain sensors for analysis of deformations of machines and devices including those used in mining techniques. FBG strain sensors have many advantages over classic strain measurements using electro resistance strain gauges. They are characterized by a significant measurement accuracy of up to $1 \mathrm{~mm}$, a service life of up to 30 years, the possibility of measuring large deformations of up to $8 \%$, significant fatigue life, the possibility of building measurement networks and, something extremely important in mining - intrinsic safety, because the operating medium is white light. The entire measurement system based on the optical interrogator was also discussed. It enables conducting both static and dynamic measurements. The results of the strain research for an engineering machine, in which the loads had exceeded $800 \mathrm{~T}$, were reported.
\end{abstract}

Keywords: optical fiber sensors with FBG fiber Bragg grating, strains, engineering machines 